\title{
Hepatic Fibrosis and Carcinogenesis in $\alpha 1-$ Antitrypsin Deficiency: A Prototype for Chronic Tissue Damage in Gain-of-Function Disorders
}

\author{
David H. Perlmutter and Gary A. Silverman \\ Departments of Pediatrics, Cell Biology, and Physiology, University of Pittsburgh School of Medicine, \\ Children's Hospital of Pittsburgh and Magee-Womens Hospital of UPMC, Pittsburgh, \\ Pennsylvania 15224 \\ Correspondence: david.perImutter@chp.edu
}

\begin{abstract}
In $\alpha 1$-antitrypsin (AT) deficiency, a point mutation renders a hepatic secretory glycoprotein prone to misfolding and polymerization. The mutant protein accumulates in the endoplasmic reticulum of liver cells and causes hepatic fibrosis and hepatocellular carcinoma by a gain-of-function mechanism. Genetic and/or environmental modifiers determine whether an affected homozygote is susceptible to hepatic fibrosis/carcinoma. Two types of proteostasis mechanisms for such modifiers have been postulated: variation in the function of intracellular degradative mechanisms and/or variation in the signal transduction pathways that are activated to protect the cell from protein mislocalization and/or aggregation. In recent studies we found that carbamazepine, a drug that has been used safely as an anticonvulsant and mood stabilizer, reduces the hepatic load of mutant AT and hepatic fibrosis in a mouse model by enhancing autophagic disposal of this mutant protein. These results provide evidence that pharmacological manipulation of endogenous proteostasis mechanisms is an appealing strategy for chemoprophylaxis in disorders involving gain-of-function mechanisms.
\end{abstract}

$T^{\text {he }}$ he classical form of $\alpha 1$-antitrypsin (AT) deficiency is a relatively common genetic disease that causes hepatic fibrosis and carcinogenesis by a gain-of-toxic function mechanism. This disorder is the most common genetic liver disease of childhood and a much more frequent cause of liver disease in adults than previously recognized (Perlmutter 2002). From work that has been done since this disorder was discovered in 1963, we now know that this liver disease results from the accumulation of mutant AT inside of liver cells. Ordinarily, wild-type AT is secreted by liver cells into the blood so that it can circulate to body fluids where it carries out its primary function, inhibition of neutrophil elastase and perhaps several other serine proteases released by neutrophils. AT is the archetype of a family of proteins that have been

Editors: Richard Morimoto, Jeffrey Kelly, and Dennis Selkoe

Additional Perspectives on Protein Homeostasis available at www.cshperspectives.org

Copyright (C) 2011 Cold Spring Harbor Laboratory Press; all rights reserved; doi: 10.1101/cshperspect.a005801

Cite this article as Cold Spring Harb Perspect Biol 2011;3:a005801 
called SERPINS because most of the members are serine protease inhibitors and share a relatively distinct mechanism for inactivating proteases (Silverman et al. 2010; Whisstock et al. 2010). In the classical deficiency state, a point mutation leads to alteration in folding early in the secretory pathway and renders the mutant protein, ATZ, prone to polymerization and aggregation. The mutant ATZ is relatively ineffective in traversing the secretory pathway with $\sim 10 \%-15 \%$ of newly synthesized molecules reaching the extracellular fluid and the remainder accumulating in the endoplasmic reticulum (ER) of hepatocytes. Chronic hepatic fibrosis/cirrhosis and carcinogenesis is caused by the cellular sequellae from accumulation of the polymerized/aggregated mutant protein in the ER by a gain-of-toxic function mechanism (Perlmutter et al. 2007).

One of the most striking aspects of this disorder is the variation in incidence and severity of the liver disease among homozygotes. A unique unbiased cohort study performed over 40 years after identifying homozygotes from a newborn screening in Sweden has shown that only $8 \%-10 \%$ develop severe liver disease (Piitulainen et al. 2005). This means that genetic and/or environmental modifiers determine whether a homozygote is susceptible to, or protected from, liver disease. We have long postulated that these modifiers affect components of the protein homeostasis machinery (Perlmutter 2002).

In this review, we will discuss what is known about the gain-of-toxic function mechanism that is responsible for liver damage in AT deficiency and focus on at least four features that are prototypic of the gain-of-function disorders associated with other aggregation-prone proteins. First, damage occurs in a tissue, which is the site of synthesis of a misfolded, mislocalized, and/or aggregation-prone protein. Second, protein homeostasis pathways, such as the proteasomal pathway, the autophagic and unfolded protein response, are activated in that tissue. Third, regulation and activity of these proteostasis mechanisms are likely sites of action for modifiers that determine variation in the incidence and severity of the clinical phenotype. Fourth, in many of the gain-of-function diseases there is evidence that mitochondrial dysfunction plays a key role in the tissue damage.

Lastly, we will discuss what is known about the other member of the SERPIN family with respect to mutations that lead to tissue damage by gain-of-function. Most of these occur less commonly and have not been as extensively investigated but have contributed to our understanding of gain-of-function mechanisms for human disease.

\section{AT DEFICIENCY: ROLE OF PROTEIN AGGREGATION IN TISSUE DAMAGE}

The classical form of AT deficiency is characterized by an autosomal codominant pattern of inheritance and affects 1 in 2000 to 3000 live births in most populations. AT is detected in the serum but it is reduced to $10 \%-15 \%$ of the normal serum levels. A point mutation leads to substitution of lysine for glutamate at residue 342 and results in abnormal folding of the mutant protein, termed ATZ, in the earliest steps of the secretory pathway. The mutant protein also has an increased tendency to polymerize, forming insoluble aggregates within the rough endoplasmic reticulum and accounting for the histological hallmark of the disease, periodic acid-Schiff-positive, diastase-resistant globules in hepatocytes (reviewed in Perlmutter 2002).

Studies by Lomas et al. showed for the first time that the ATZ protein was prone to polymerization and aggregation (Lomas et al. 1992). A recent study by Yamasaki et al. which elucidated the crystal structure of a stable serpin dimer, has provided the basis for an interesting new model in which a domain swapping mechanism explains the formation of polymers and insoluble aggregates of ATZ (Yamasaki et al. 2008). The model (Fig. 1) predicts that the final step in the folding of the serpin molecule is the incorporation of strand $5 \mathrm{a}$ (s5a) into the central $\beta$ sheet $A$, while leaving the contiguous reactive center loop (RCL) and future s4a perched upon the top of the molecule where it serves as bait for target peptidases (reviewed in Whisstock et al. 2000; Silverman et al. 2010). Because the 


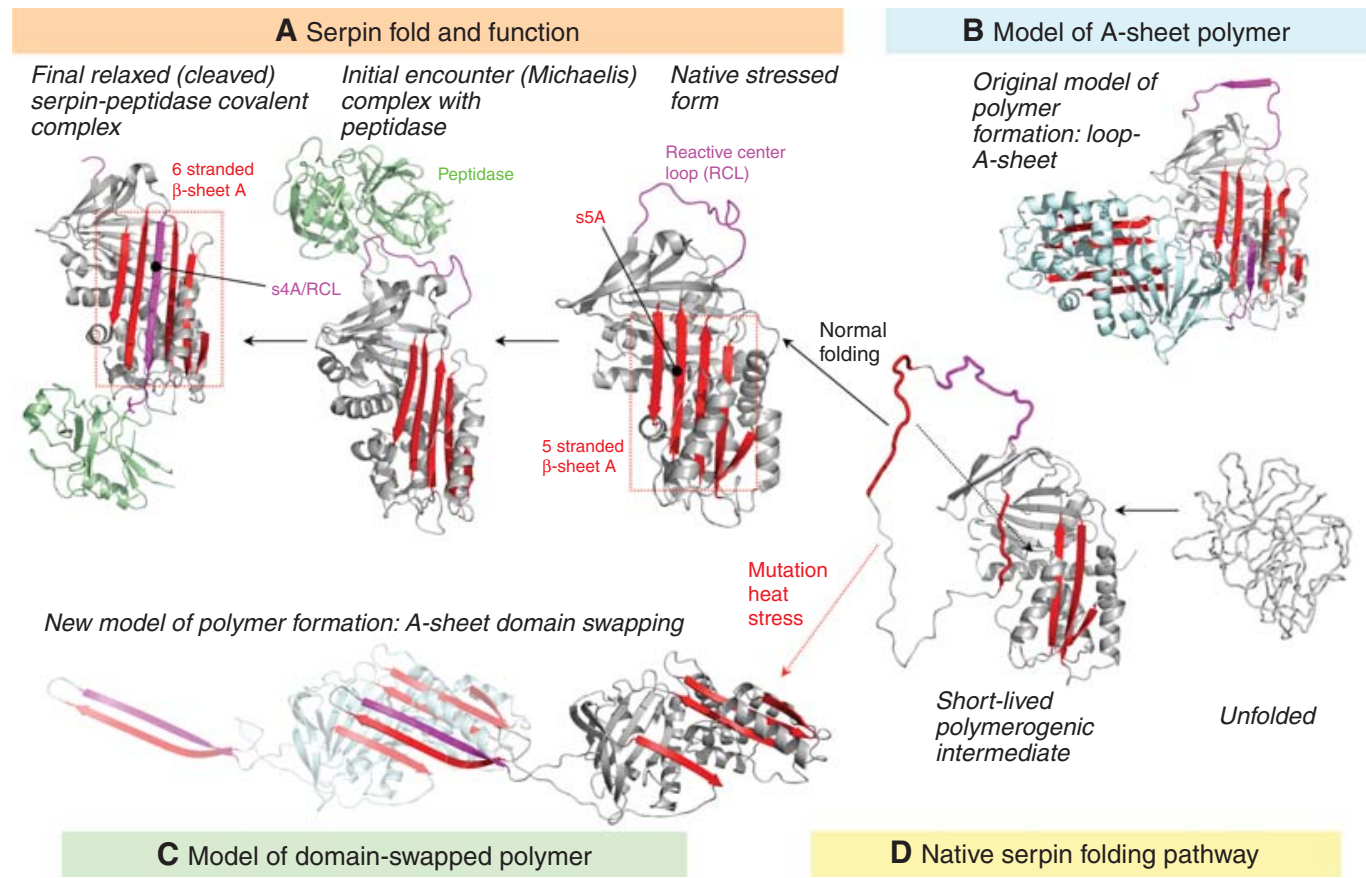

Figure 1. Serpin folding and misfolding. (A) Active serpins fold into a metastable state. Binding of target protease results in RCL cleavage. The serpin undergoes a radical conformational change (RCL/s4A incorporation into $\alpha$-sheet A) that culminates in protease inhibition via distortion of the catalytic residues. (B) Loop-sheet model of serpin polymerization. The RCL of one molecule is inserted into the open $\alpha$-sheet A of another. $(C)$ Domain-swapped model of serpin polymerization. The model is based on the structure of a domainswapped AT dimer, where s5A and s4A (RCL) of a donor molecule insert into the $\alpha$-sheet A of a recipient. (D) Normal serpins may fold through a polymerogenic intermediate that is stabilized by certain mutations. Black dashed arrow indicates gap in $\alpha$-sheet A that accommodates s5A to form the s4A (RCL) exposed native form in $(A)$ or the s5A and s4A domain-swapped structure in $(C)$. (Reproduced from Whisstock et al. 2010 and printed with permission from the American Society for Biochemistry and Molecular Biology (C) 2010.)

substitution of lysine for glutamate 342 that characterizes the ATZ molecule lies within s5a and because this substitution disrupts the usual intramolecular interactions with lysine 290 on s6a and threonine 203, folding is slowed and yields a more stable intermediate without inclusion of $s 5 \mathrm{a}$. The exposed $s 5 \mathrm{a}$ and contiguous RCL form a loop that inserts into the $\alpha$-sheet A gap present in a second partially unfolded ATZ molecule, thereby forming a more stable six-stranded $\alpha$-sheet structure. This insertion is analogous to that observed in the cleaved or latent conformations of AT in which the RCL loop has been inserted into sheet A. Although the domain swapped structure is that of a self-terminating dimer, molecular modeling using the partially unfolded monomers in a head-to-tail orientation predicts formation of a linear polymer in which the exposed s5a-RCL loop from the second molecule inserts into the $\alpha$-sheet A gap of a third ATZ molecule, and so on. Finally, domain swapping requires that helix I, which connects s6A to s5a, remains uncoiled. Because this uncoiled domain is $\sim 50 \%$ hydrophobic, this region may facilitate lateral associations among linear polymers, and enhance the formation of the tangled aggregates seen in the ER of liver cells in AT deficiency (Yamasaki et al. 2008). A delay in proper folding of the ATZ variant, rather than a complete failure in folding, provides an explanation for how a small number $(10 \%-15 \%)$ of these molecules are kinetically capable of reaching a conformation that allows traversal of the secretory pathway, 
whereas the majority of mutant ATZ proteins accumulate as polymers and insoluble aggregates within the ER. This domain swapping mechanism also provides an explanation for why polymerized ATZ in the ER does not activate the unfolded protein response, as the structure of the polymer closely resembles relaxed and latent conformations that can be acquired by properly folded serpin monomers.

In most cases, the liver disease associated with AT deficiency is slowly progressing and characterized by fibrosis with low-grade inflammation. The intrahepatic accumulation of ATZ also predisposes to dysplasia, adenomas, and carcinomas (Eriksson et al. 1986). The accumulation of mutant ATZ in the ER accounts for the periodic acid-Schiff-positive, diastase-resistant globules in hepatocytes. Compelling evidence for a gain-of-toxic function mechanism of liver damage comes from the observation that mice transgenic for the mutant human ATZ gene develop liver disease (Dycaico et al. 1988; Carlson et al. 1989). The liver disease that develops in one of the transgenic models, the PiZ mouse, has been more extensively characterized in recent years and recapitulates many of the features of the disease that occurs in humans. In addition to the intrahepatocytic globules, there is slowly progressing fibrosis, low-grade inflammation and regeneration, mild steatosis, dysplasia, and carcinomatosis. Furthermore, the severity of this pathology varies with the background strain of the mouse (D.H. Perlmutter, unpubl.). These mice have normal levels of endogenous antiproteases and so this liver disease must be attributable to the gain-of-toxic function mechanism.

\section{AT DEFICIENCY: ROLE OF MITOCHONDRIAL DYSFUNCTION IN LIVER DISEASE}

The molecular basis for liver cell injury in AT deficiency is still largely undefined. Detailed studies of liver pathology in the PiZ mouse model showed significant increases in mitochondrial damage and mitochondrial injury together with caspase-3 activation in situ (Teckman et al. 2004). Similar alterations were noted in the liver of AT-deficient patients (Teckman et al. 2004). Mitochondrial depolarization followed induction of ATZ expression in a cell line model. Most importantly, cyclosporine A treatment of PiZ mice reduced mitochondrial damage and mortality after the stress of starvation (Teckman et al. 2004), providing further evidence for the concept that mitochondrial dysfunction is a sequelae of the primary cellular defect in AT deficiency.

BrdU labeling studies in the PiZ mouse model have permitted some observations about hepatocellular proliferation in AT deficiency (Rudnick et al. 2004). The results indicate that there is increased proliferation but almost exclusively in globule-devoid hepatocytes. Consistent with this, most, if not all, of the adenomas and carcinomas that occur in the human disease arise in the globule-devoid cells (reviewed in Rudnick and Perlmutter 2005; Perlmutter 2006). It is not clear how these globule-devoid hepatocytes arise but they are known to have less of the polymerized/aggregated ATZ than globule-containing hepatocytes (An et al. 2005). We have speculated that the globule-devoid hepatocytes are younger and have a selective proliferative advantage when in the presence of globule-containing hepatocytes. The globule-containing cells have more aggregated ATZ and mitochondrial dysfunction, which renders them relatively impaired in cell proliferation. Furthermore, the degree of regenerative activity in the liver is directly proportional to the number of these globulecontaining hepatocytes. Thus, we have theorized that the globule-containing hepatocytes are "sick but not dead" and are responsible for regenerative signaling but the globule-devoid hepatocytes are the predominant ones that can respond to these signals. This cross talk presumably perpetuates hepatocellular proliferation in the presence of inflammation, creating a milieu that is prone to carcinogenesis.

\section{AT DEFICIENCY: DETERMINANTS OF TISSUE-SPECIFIC DAMAGE}

AT deficiency is also known as a cause of prematurely developing chronic obstructive pulmonary disease (COPD)/emphysema. In contrast 
to the pathogenesis of liver disease, the mechanism of lung damage has long been thought to involve loss-of-function (Crystal 1990). The elastase-antielastase theory for the pathogenesis of COPD is based on the concept that in the absence of sufficient AT, neutrophil elastase, and perhaps other neutrophil proteases, progressively destroy the connective tissue matrix of the lung. In the classical form of AT deficiency this is because of the reduction in AT levels in the circulating blood as well as in body fluids and tissues such as lung. This is the basis for replacement therapy with purified AT that is administered by intravenous infusion or by inhalation therapy to AT-deficient patients with established COPD.

However, several observations have suggested that gain-of-toxic function mechanisms may contribute to lung injury. For example, ATZ polymers deposited in the lung from the extracellular fluid may attract neutrophil and neutrophilic inflammatory effectors that probably increase the severity of lung damage (Mahadeva et al. 2005). Gain-of-toxic function could also theoretically result from the effects of mutant ATZ expression in bronchoalveolar macrophages and respiratory epithelial cells in the same way it happens in hepatocytes $(\mathrm{Hu}$ and Perlmutter 2002). These effects could explain the fact that replacement therapy has had relatively limited clinical efficacy to date.

\section{AT DEFICIENCY: VARIATION IN CLINICAL DISEASE AMONG HOMOZYGOTES IS BECAUSE OF GENETIC AND/OR ENVIRONMENTAL MODIFIERS}

In the only unbiased clinical epidemiological study of AT deficiency Sveger has shown that only $8 \%$ of homozygotes for the classical form of AT deficiency develop clinically significant liver disease over the first three decades of life (Piitulainen et al. 2005). The results of this study have led to the concept that modifiers, either genetic or environmental, predispose a subgroup of homozygotes to liver disease and/or protect the remainder of the population from liver disease. Furthermore, we have theorized that these putative modifiers affect the pathways responsible for disposal of mutant ATZ and/or the protective signaling pathways that are activated by accumulation of ATZ in the ER (Perlmutter et al. 2007). This theory envisions "secondary" alterations that are subtle, in the sense that they are clinically silent when inherited or acquired by a host that has not inherited the mutation of ATZ. The modifiers are likely to be heterogeneous among patients and their families because this would explain the rather remarkable diversity in natural history of liver disease that has been observed. In many ways, this theory is based on the concept that subtle alterations in the protein homeostasis network represent modifiers of cell and tissue damage that is caused by aggregated proteins through gain-of-toxic function (Fig. 2). One study has provided substantiation for this theory by showing a delay in intracellular disposal of ATZ after gene transfer into cell lines from homozygotes with known liver disease compared to cell lines from homozygotes that had no evidence of liver disease ( Wu et al. 1994).

\section{AT DEFICIENCY: ROLE OF PROTEASOMAL AND AUTOPHAGIC PATHWAYS AS MODIFIERS OF TISSUE DAMAGE}

We hypothesized that pathways responsible for intracellular degradation of mutant ATZ that accumulate in the ER would be one category of protein homeostasis mechanisms that are altered by genetic and/or environmental modifiers. To address this hypothesis, we and others sought to determine the mechanisms by which ATZ is degraded when it accumulates in the ER. Early studies in both yeast and mammalian cell lines showed that the proteasome participates in degradation of mutant ATZ (Qu et al. 1996; Werner et al. 1996; Teckman et al. 2001). In fact, degradation of mutant ATZ by the proteasome was one of the original observations that led to the recognition of the ER-associated degradation (ERAD) pathway. ERAD is a pathway by which proteins in the lumen or membrane of the ER are targeted for degradation by retrograde translocation into cytoplasm where ubiquitination and proteasomal degradation 
D.H. Perlmutter and G.A. Silverman
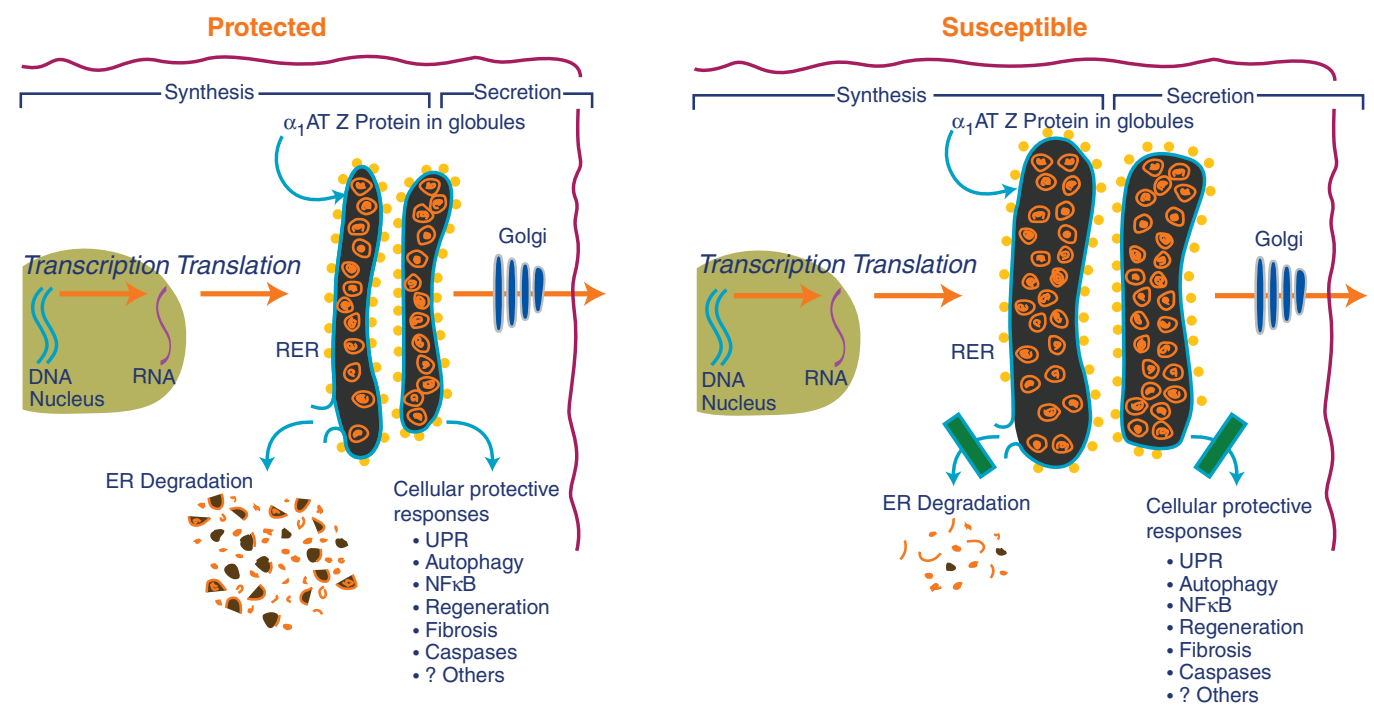

Figure 2. Cellular factors that determine whether an AT-deficient individual is protected or susceptible to liver disease. In the susceptible host, there is greater accumulation of insoluble ATZ in the ER because of subtle alterations in putative proteostasis network regulatory mechanisms. Here these proteostasis regulatory mechanisms are envisioned as either ER degradation pathways or cellular protective responses.

take place (reviewed in Brodsky and Wojcikiewicz 2009). We know now that there are different types of ERAD for luminal and membrane proteins and there may even be further subspecialization for different types of luminal proteins but the exact chaperones and proteins that recognize ATZ and mediate its transport into the cytoplasm are not yet elucidated. Studies using a cell-free microsomal translocation system suggest that ATZ degradation by the proteasome occurs by both ubiquitindependent and ubiquitin-independent mechanisms (Teckman et al. 2000).

Nevertheless, there was evidence from these studies of the proteasomal pathway in mammalian cell lines, cell-free microsomal translocation systems, and yeast that this pathway could not completely account for disposal of ATZ. Autophagy was first implicated in AT deficiency when a marked increased in autophagosomes were observed in fibroblast cell lines engineered for expression of ATZ (Teckman and Perlmutter 2000). Increased autophagosomes were observed in the liver of PiZ mice and in liver biopsy specimens from patients (Teckman and Perlmutter 2000).
Autophagy is a catabolic process by which the cell digests its internal constituents to generate amino acids in response to nutrient starvation and other stress states. It also appears to play a role in homeostasis, cell growth, and differentiation. It begins with the formation of a membranous platform around a targeted region of the cell. This platform becomes a double-membrane vesicle as it envelopes cytoplasm together with parts of or entire subcellular organelles. Eventually this autophagosome fuses with the lysosome for degradation of its contents.

Thus, the presence of abundant autophagosomes in the liver in AT deficiency and its models raised the possibility that autophagy could be involved in degrading the mutant ATZ that is retained in the ER. Indeed the initial study went on to show that disposal of ATZ was partially abrogated by chemical inhibitors of autophagy, including 3-methyladenine, wortmannin and LY-294002 (Teckman and Perlmutter 2000). However, because these drugs have other cellular effects and therein cannot be considered specific for autophagy we sought to provide genetic evidence that autophagy participated 
in disposal of ATZ. For this we engineered an embryonic fibroblast cell line (MEF) from an ATG5-null mouse for expression of ATZ (Kamimoto et al. 2006). The results showed a marked delay in degradation of ATZ in the ATG5-null compared to the wild type MEFs. Furthermore, in the ATG5-null cell line it became possible to observe massive accumulation of ATZ with very large inclusions throughout the cytoplasm. Therefore, in addition to providing definitive evidence that it contributes to the disposal of ATZ, these studies suggest that autophagy plays a "homeostatic" role in the AT-deficient state by preventing toxic cytoplasmic accumulation of ATZ through piecemeal digestion of insoluble aggregates.

A study by Kruse et al using a completely different strategy also showed the importance of autophagy in disposal of ATZ (Kruse et al. 2006a). This group expressed human ATZ in a library of yeast mutants and screened for mutants that were impaired for degradation of ATZ. One of the mutant corresponded to the yeast homolog of mammalian autophagy protein ATG6. In the absence of this ATG6 homolog or the homolog of ATG16 there was a marked delay in disposal of human ATZ. These studies were particularly revealing because delay in degradation of ATZ was most apparent in the autophagy-deficient yeast strains when ATZ was expressed at high levels. At lower levels of expression, ATZ was degraded at a rate not significantly different from that in wild type yeast. These results are consistent with the notion that at lower levels of expression ATZ in the ER is predominantly soluble and can be degraded by the proteasome whereas at higher levels of expression it is more likely to transition to insoluble polymers/aggregates that require autophagy for disposal.

Kruse et al. also discovered that a mutant subunit of fibrinogen that forms insoluble aggregates in the ER of liver cells in an inherited form of fibrinogen deficiency depends on autophagy for disposal (Kruse et al. 2006b). This type of fibrinogen deficiency has been associated with a chronic liver disease characterized by distinct fibrillar aggregates in the ER of liver cells. These results substantiate the concept that chronic liver disease can be caused by accumulation of an aggregation-prone protein in the ER and that autophagy is specialized for the disposal of aggregation-prone proteins that accumulate in the ER.

The presence of increased hepatic autophagosomes in AT deficiency could potentially indicate that there is some defect in clearance of autophagosomes rather than an increase in formation of autophagosomes. We have recently investigated this issue by monitoring LC3 isoform conversion in a cell line model with inducible expression of ATZ. The results indicate that ATZ expression is sufficient to elicit LC3 conversion and that conversion is further accentuated by the presence of lysosomal inhibitors (Hidvegi et al. 2010). Thus, at least within the context of a cell line model, accumulation of ATZ in the ER results in increased formation of autophagosomes and increased autophagic flux without a defect in clearance.

Several lines of evidence suggest that there are one or more other pathways that contribute to disposal of ATZ. A pathway that depends on a tyrosine phosphatase activity has been described (Cabral et al. 2002). The studies of Kruse et al indicated that ATZ could be transported to the trans-Golgi and then targeted to the lysosome of yeast but a comparable pathway has not been described in mammalian cells (Kruse et al. 2006a).

Modifiers that affect the function of any of these pathways could increase susceptibility to liver disease according to our theory. Indeed, a single nucleotide polymorphism (SNP) in the downstream flanking region of ER mannosidase I has been implicated in early-onset liver disease among AT-deficient individuals (Pan et al. 2009). Because ER mannosidase I plays a role in ERAD a polymorphism that affects its function would be a prime candidate for a modifier of liver disease in AT deficiency. However, further epidemiological studies are needed to determine if this SNP is truly affecting liver disease susceptibility and further cellular studies are needed to identify how it alters ATZ accumulation. A SNP in the upstream flanking region of the AT gene itself has also been implicated in liver disease susceptibility (Chappell 
et al. 2008). There is no reason to believe that this SNP would affect the disposal of ATZ. The logical explanation for the effect of this polymorphism would be to increase expression of ATZ but the published results did not substantiate that notion. Furthermore, the study could have led to an entirely different conclusion with a legitimate alternate way of classifying one of the patient groups.

\section{AT DEFICIENCY: ROLE OF CELLULAR RESPONSE PATHWAYS ACTIVATED BY ACCUMULATION OF ATZ IN THE ER}

According to our theory for pathogenesis of liver disease in AT deficiency, modifiers would also be predicted to alter the functioning of cellular signaling pathways that might be activated to protect the cell from aggregated proteins. To address this we began a series of studies designed to determine what signaling pathways were activated when ATZ accumulated in the ER. We reasoned that this would require cell line and mouse model systems with inducible rather than constitutive expression of ATZ because the latter would potentially permit adaptations that could obscure the primary signaling effects. A series of studies using these kinds of systems have shown that the autophagic response and the $\mathrm{NF \kappa B}$ signaling pathway but not the unfolded protein response are activated when ATZ accumulates in the ER (Hidvegi et al. 2005; Kamimoto et al. 2006). Activation of the autophagic response was shown by investigating the liver of a novel mouse model with hepatocyte-specific inducible expression of ATZ, the Z mouse, bred onto the GFP-LC3 mouse background. LC3 is an autophagosomal membrane-specific protein and so the GFP-LC3 mouse makes green fluorescent autophagosomes. Green fluorescent autophagosomes appear in the liver of the GFP-LC3 mouse only after 24 hours of starvation. In the Z x GFP-LC3 mouse green fluorescent autophagosomes are seen merely by allowing hepatocyte expression of the ATZ gene to be induced (Kamimoto et al. 2006). $\mathrm{GFP}^{+}$autophagosomes are not seen in the liver of the Saar x GFP-LC3 mouse, which has hepatocyte-specific inducible expression of the AT Saar variant that accumulates in the ER but does not polymerize. Thus, autophagy is activated when ATZ accumulates in the ER and the autophagic pathway then plays a critical role in disposal of ATZ and preventing massive intracellular aggregates.

Activation of NFKB is another hallmark of the cellular response to ATZ accumulation (Hidvegi et al. 2005). One of the most interesting aspects of the NFKB signaling pathway under these circumstances is that it is associated with a rather limited set of downstream transcriptional targets (Hidvegi et al. 2007). Indeed the most significant change in expression that could be attributable to NFKB is down-regulation of Egr-1, a transcription factor that is essential for hepatocyte proliferation and the hepatic regenerative response (Liao et al. 2004). Our most recent studies have indicated that the down-regulation of Egr-1 in the liver of the $\mathrm{Z}$ mouse when ATZ expression is induced is directly attributable to the action of NFKB (A. Mukherjee, D.H. Perlmutter, unpubl.). Furthermore, the complex of proteins that assembles to form NFKB when ATZ accumulates in the ER has a profile that is entirely distinct from that which forms when cells are treated with TNF or tunicamycin (A. Mukherjee and D.H. Perlmutter, unpubl.). Finally, mating of the PiZ mouse to a mouse model with conditional hepatocyte-specific deficiency of NFкB activity shows more severe inflammation, fibrosis, steatosis, dysplasia and more hepatocytes with globules (A. Mukherjee, T. Hidvegi, D.H. Perlmutter, unpubl.), indicating that $\mathrm{NF \kappa B}$ signaling is intended to protect the liver from the effects of ATZ accumulation. Together, the data on NFкB suggest that it plays a particularly important role in the effects of ATZ on cell proliferation, survival and ultimately the predisposition to hepatic carcinogenesis in $\mathrm{AT}$ deficiency.

The absence of the unfolded protein response (UPR) is also noteworthy (Hidvegi et al. 2005; Hidvegi et al. 2007). For one, it means that activation of autophagy and NFкB in cells which accumulate mutant ATZ is independent of the UPR, another distinct characteristic of the cellular response in the AT deficiency 
state. With what is known about the mechanism by which the UPR is initiated it has always been relatively easy to understand how polymerized and aggregated would not elicit the UPR. Results of the recent structural studies by Huntington and colleagues provide an explanation for why soluble monomeric ATZ does not get recognized by the UPR apparatus. Those results suggest that the monomeric ATZ intermediate adopts a conformation that resembles the wild type molecule and therein would not be recognized as unfolded (Yamasaki et al. 2008).

Genomic analysis of liver from the $\mathrm{Z}$ mouse has identified other changes in gene expression that are attributable to the cellular response to ATZ (Hidvegi et al. 2007). One of these changes, up-regulation of regulator of $\mathrm{G}$ signaling 16 (RGS 16), may represent a mechanism by which autophagy is activated in the liver in AT deficiency. We have found that the RGS16 response is characteristic and specific for ATZ. Because RGS16 antagonizes Goi3 and Gai3 plays a role in inhibiting hepatic autophagy (Gohla et al. 2007), we have hypothesized that increased RGS16 when ATZ accumulates in the ER leads to reversal of the inhibition of autophagy that would otherwise pertain in resting hepatocytes. There is still relatively limited information about how RGS16 is up-regulated and where in the cell it acts to antagonize Goi3.

\section{AT DEFICIENCY: NOVEL THERAPEUTIC STRATEGIES BASED ON GAIN-OF-TOXIC FUNCTION MECHANISMS}

The autophagic response would appear to be an ideal target for potential therapies because it plays a role in disposal of mutant ATZ, is particularly specialized for disposal of the insoluble aggregated forms of ATZ that are integral to the pathogenesis of hepatic fibrosis and carcinomatosis, and because the autophagic response is specifically activated by accumulation of ATZ in the ER. For this reason, we recently investigated one of a list of FDA-approved drugs that are known to enhance autophagy. Carbamazepine (CBZ) was selected because it has the most extensive safety profile, having been used for years as an anticonvulsant and mood stabilizer. We found that this drug enhanced the autophagic disposal of ATZ in cell line and mouse models of AT deficiency (Fig. 3) (Hidvegi et al. 2010). Furthermore, CBZ reduced the hepatic load of ATZ and hepatic fibrosis in vivo in the mouse model (Hidvegi et al. 2010). These results indicate that CBZ can be tested immediately in clinical trials for treatment of AT deficiency-associated liver disease and provide a proof-in-principle for the therapeutic use of autophagy enhancers. This concept has been further substantiated by recent results from high-content screening of drug libraries using a novel C. elegans model of AT deficiency. In a pilot screen, 4 of 6 hit compounds are known to be autophagy enhancers (Gosai et al. 2010). These results are also particularly exciting because they provide evidence for the concept that the endogenous protein homeostasis machinery can be used to prevent tissue damage from mutant proteins.

Ultimately, it would be ideal to investigate these drugs together with small molecules that alter the conformation of the mutant ATZ in such a way that insoluble ATZ is destroyed in the liver and soluble ATZ is secreted, thereby preventing both liver and lung disease in AT deficiency. The recently described domainswapping mechanism for polymerization of ATZ (Yamasaki et al. 2008) provides a basis for believing that only relatively minor changes in the kinetics of folding and degradation would be needed to facilitate secretion of the soluble forms of ATZ.

\section{OTHER SERPINOPATHIES THAT CAUSE HUMAN DISEASE BY GAIN-OF-FUNCTION MECHANISMS}

Although mutations in AT have been the most widely studied, missense mutations in other serpin family members are increasingly recognized as causes of human disease. Most of these mutations cluster in three key regions of the serpin scaffold; the shutter region, the proximal hinge region and the distal hinge region (Stein and Carrell 1995). The proximal and distal hinge regions help maintain the RCL in its active conformation above the body of the 
D.H. Perlmutter and G.A. Silverman

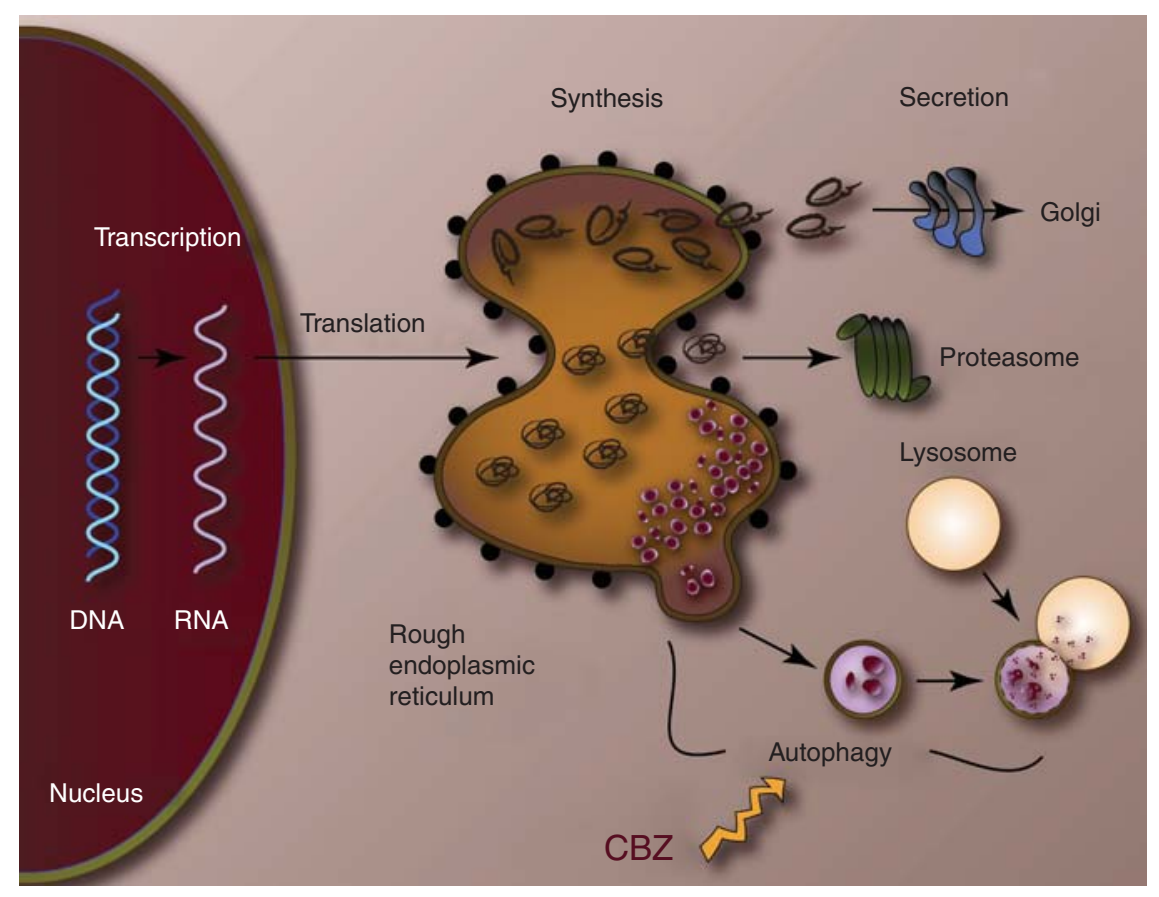

Figure 3. Cellular action of carbamazepine (CBZ). CBZ enhances autophagic disposal of insoluble ATZ (red globules). Soluble ATZ may be secreted (top) or transported by ERAD for degradation by the proteasome.

serpin where it is available for peptidase binding. These regions are also important for carrying out the serpin inhibitory mechanism by facilitating the movement and insertion of the proximal portion of the RCL into the strand 4 position of $\alpha$-sheet A (Huntington et al. 2000). This RCL movement translocates the bound peptidase and traps it as a stable covalent intermediate (Huntington et al. 2000). The shutter region is comprised of several structural elements, which regulate the sliding of $\alpha$-sheet A strands to allow for insertion of the RCL after peptidase cleavage. Mutations in these regions result in serpin dimerization and higher order polymerization by domain swapping as described above for ATZ or possibly by a loopsheet insertion (Fig. 1) (Lomas et al. 1992; Yamasaki et al. 2008). In the loop-sheet insertion model, the serpin folds normally, but destabilizing mutations allow for premature opening of the A-sheet and insertion of the intact RCL from the same (latent conformation) or from a different serpin molecule (loop-A-sheet A dimer). Latent serpins can also polymerize as the RCL of a serpin binds to the position in $\alpha$-sheet $C$ vacated by the distal hinge movement required to form the latent molecule (loop-Csheet dimer).

At least five different serpins causing human diseases have been found with mutations in one of the three critical regions, but most cluster in the shutter region (Stein and Carrell 1995; Gooptu and Lomas 2009). Heterozygous mutations (L55P or P229A) in $\alpha 1$-antichymotrypsin, an inhibitor of cathepsin $G$ and mast cell chymase, can result in misfolded protein accumulation in the liver and chronic obstructive pulmonary disease analogous to that observed with AT deficiency (Faber et al. 1993; Poller et al. 1993). A homozygous mutation of heparin cofactor II (E428K), an inhibitor of thrombin, was associated with a pro-thrombotic state, especially in the presence of a heterozygous antithrombin deficiency (Villa et al. 1999; Corral et al. 2004a). This mutation, which is identical to that of ATZ, impaired mutant protein secretion to circulating levels that were $\sim 5 \%$ of normal. An autosomal dominant 
mutation of $\mathrm{C} 1$ inhibitor, which regulates the classical complement and bradykinin cascades, is the cause of hereditary angioedema. Several of the mutations (A436T, V451M, F455S, and P476S) lead to impaired secretion, intracellular degradation, and polymerization (Aulak et al. 1993; Eldering et al. 1995; Verpy et al. 1995). Autosomal dominant mutations in antithrombin lead to thrombosis. Although many of these mutations lead to misfolding and impaired secretion, others lead to the formation of latent species in the circulation (Bruce et al. 1994; Zhou et al. 1999; Corral et al. 2004b). The latent conformation is caused by instability of native structure, which allows for insertion of the intact RCL into $\beta$-sheet $A$ and displacement of s1C. Not only are latent molecules unable to inhibit thrombin, they form inactive dimers with wild-type proteins via $\alpha$-stand linkages as loop-C-sheet dimers as described above (Bruce et al. 1994; Zhou et al. 1999; Corral et al. 2004b). Thus, circulating mutant proteins in the latent conformation predispose to thrombosis by further decreasing the concentration of active inhibitor.

In contrast to AT deficiency associated with shutter region mutations, those in the hepatically synthesized proteins heparin cofactor II, C1 inhibitor, and antithrombin do not appear to cause liver disease and intracellular inclusions despite causing protein misfolding, polymerization, and impaired secretion. In part, this difference may be caused by a concentration effect, as the normal hepatic synthesis of these latter proteins is only approximately $10 \%$ that of AT (Gooptu and Lomas 2009).

Mutations in the neuroserpin gene are the cause of an autosomal dominant neurodegenerative disorder characterized by progressive deterioration of cognition, memory, and visuospatial skills (Davis et al. 1999a; Davis et al. 1999b). Some patients also develop progressive myoclonus epilepsy (PME) (Takao et al. 2000). Histopathological examination of affected individuals show the accumulation of PAS positive, diastase resistant intracellular inclusion bodies within neurons located in the deeper layers of the cerebral cortex and subcortical nuclei, including the substantia nigra (Davis et al. 1999a; Davis et al. 1999b). In cases with PME, neurons in the cerebellum and the cerebellar dentate nucleus are also involved (Davis et al. 2002). These inclusions (Collins bodies) are comprised almost exclusively of misfolded and/or polymerized neuroserpin and are located within dilated cisternae of the rough endoplasmic reticulum (Davis et al. 1999a; Davis et al. 1999b). Hence, this disorder has been described as familial encephalopathy with neuroserpin inclusion bodies (FENIB) (Davis et al. 1999a; Davis et al. 1999b). At least five different missense neuroserpin mutations have been identified, and they all involve the shutter region. Based on the nature of the amino acid substitutions and their location within the serpin scaffold, these mutations are predicted to confer different degrees of instability relative to the native fold (Davis et al. 2002). Indeed, the more destabilizing mutations (G392R> G392E $>$ H338R $>$ S52R $>$ S49P) are associated with a greater number and more pervasive distribution of Collins bodies and an earlier onset and a more severe progression of dementia and PME (Davis et al. 2002; Gooptu and Lomas 2009). This correlation was supported by transient transfection of the mutant genes into cell lines, and showing that the more destabilizing mutations resulted in the formation of longer polymers and a greater degree of ER retention (Miranda et al. 2004; Miranda et al. 2008).

Because of their metastable active conformation, all wild-type serpins are capable of polymerizing in vitro in a concentration and temperature dependent process (Dafforn et al. 1999). Under certain conditions, this process appears to occur in vivo. Megsin (SERPINB7) is an intracellular serpin that is expressed in the mesangial cells within the glomerulus of the kidney (Miyata et al. 1998). In IgA and diabetic nephropathy, this serpin is up-regulated and appears to drive mesangial cell proliferation and mesangial matrix deposition leading to glomerulosclerosis (Suzuki et al. 1999). Transgenic mice over expressing human megsin also develop glomerular sclerosis (Miyata et al. 2002). However, transgenic rats expressing the same transgene develop intracellular PAS inclusion bodies within glomerular epithelial, tubular 
(proximal and distal), and collecting duct cells (Inagi et al. 2005). The PAS inclusions were positive for megsin by immunohistochemistry. Similar inclusions were found in pancreatic acinar cells and islet $\alpha$ cells. These animals developed progressive renal failure and diabetes and die within about 10 weeks. Because many serpins are acute phase reactants, these data suggest that inflammatory reactions that drive serpin over expression may be complicated by the deleterious consequences of serpin polymerization.

There is still relatively limited information about the role of protein homeostasis pathways in tissue injury that occurs in these other serpinopathies and whether variations in the function of endogenous proteostasis pathways determine the incidence and severity of tissue injury. We suspect that the principles affecting the classical form of AT deficiency will also apply to these disorders. Variation in the function of proteostasis pathways would be a likely mechanism for genetic and environmental modifiers of these disorders. Furthermore, strategies that target the proteostasis pathways may be attractive for therapy as well as prevention of cell and organ damage.

\section{REFERENCES}

An JK, Blomenkamp K, Lindblad D, Teckman JH. 2005. Quantitative isolation of alphalAT mutant $\mathrm{Z}$ protein polymers from human and mouse livers and the effect of heat. Hepatology 41: 160-167.

Aulak KS, Eldering E, Hack CE, Lubbers YP, Harrison RA, Mast A, Cicardi M, Davis AEd. 1993. A hinge region mutation in C1-inhibitor (Ala436->Thr) results in nonsubstrate-like behavior and in polymerization of the molecule. J Biol Chem 268: 18088-18094.

Brodsky JL, Wojcikiewicz RJ. 2009. Substrate-specific mediators of ER associated degradation (ERAD). Curr Opin Cell Biol 21: 516-521.

Bruce D, Perry DJ, Borg JY, Carrell RW, Wardell MR. 1994. Thromboembolic disease due to thermolabile conformational changes of antithrombin Rouen-VI (187 Asn-> Asp). J Clin Invest 94: 2265-2274.

Cabral CM, Liu Y, Moremen KW, Sifers RN. 2002. Organizational diversity among distinct glycoprotein endoplasmic reticulum-associated degradation programs. Mol Biol Cell 13: 2639-2650.

Carlson JA, Rogers BB, Sifers RN, Finegold MJ, Clift SM, DeMayo FJ, Bullock DW, Woo SL. 1989. Accumulation of PiZ alpha 1-antitrypsin causes liver damage in transgenic mice. J Clin Invest 83: 1183-11890.
Chappell S, Hadzic N, Stockley R, Guetta-Baranes T, Morgan K, Kalsheker N. 2008. A polymorphism of the alpha 1 -antitrypsin gene represents a risk factor for liver disease. Hepatology 47: 127-132.

Corral J, Aznar J, Gonzalez-Conejero R, Villa P, Minano A, Vaya A, Carrell RW, Huntington JA, Vicente V. 2004a. Homozygous deficiency of heparin cofactor II: relevance of P17 glutamate residue in serpins, relationship with conformational diseases, and role in thrombosis. Circulation 110: 1303-1307.

Corral J, Huntington JA, Gonzalez-Conejero R, Mushunje A, Navarro M, Marco P, Vicente V, Carrell RW. 2004b. Mutations in the shutter region of antithrombin result in formation of disulfide-linked dimers and severe venous thrombosis. J Thromb Haemost 2: 931-939.

Crystal RG. 1990. aAlpha 1-Antitrypsin deficiency, emphysema, and liver disease. Genetic basis and strategies for therapy. J Clin Invest 85: 1343-1352.

Dafforn TR, Mahadeva R, Elliott PR, Sivasothy P, Lomas DA. 1999. A kinetic mechanism for the polymerization of alpha 1-antitrypsin. J Biol Chem 274: 9548-9555.

Davis RL, Holohan PD, Shrimpton AE, Tatum AH, Daucher J, Collins GH, Todd R, Bradshaw C, Kent P, Feiglin D, et al. 1999a. Familial encephalopathy with neuroserpin inclusion bodies. Am J Pathol 155: 1901-1913.

Davis RL, Shrimpton AE, Carrell RW, Lomas DA, Gerhard L, Baumann B, Lawrence DA, Yepes M, Kim TS, Ghetti B, et al. 2002. Association between conformational mutations in neuroserpin and onset and severity of dementia. Lancet 359: 2242-2247.

Davis RL, Shrimpton AE, Holohan PD, Bradshaw C, Feiglin D, Collins GH, Sonderegger P, Kinter J, Becker LM, Lacbawan F, et al. 1999b. Familial dementia caused by polymerization of mutant neuroserpin. Nature 401: 376-379.

Dycaico MJ, Grant SG, Felts K, Nichols WS, Geller SA, Hager JH, Pollard AJ, Kohler SW, Short HP, Jirik FR, et al. 1988. Neonatal hepatitis induced by alpha 1-antitrypsin: a transgenic mouse model. Science 242: 14091412.

Eldering E, Verpy E, Roem D, Meo T, Tosi M. 1995. COOHterminal substitutions in the serpin $\mathrm{C} 1$ inhibitor that cause loop overinsertion and subsequent multimerization. J Biol Chem 270: 2579-2587.

Eriksson S, Carlson J, Velez R. 1986. Risk of cirrhosis and primary liver cancer in alpha 1-antitrypsin deficiency. N Engl J Med 314: 736-739.

Faber JP, Poller W, Olek K, Baumann U, Carlson J, Lindmark B, Eriksson S. 1993. The molecular basis of alpha 1antichymotrypsin deficiency in a heterozygote with liver and lung disease. J Hepatol 18: 313-321.

Gohla A, Klement K, Piekorz RP, Pexa K, vom Dahl S, Spicher K, Dreval V, Haussinger D, Birnbaumer L, Nurnberg B. 2007. An obligatory requirement for the heterotrimeric $\mathrm{G}$ protein $\mathrm{Gi} 3$ in the antiautophagic action of insulin in the liver. Proc Natl Acad Sci 104: 3003-3008.

Gosai SJ, Kwak JH, Luke CJ, Long OS, King DE, Kovatch KJ, Johnston PA, Shun TY, Lazo JS, Perlmutter DH, et al. 2010. Automated high-content live animal drug screening using C. elegans expressing the aggregation-prone serpin alpha-1-antitrypsin Z. PLoS One 5: e15460. 
Gooptu B, Lomas DA. 2009. Conformational pathology of the serpins: themes, variations, and therapeutic strategies. Annu Rev Biochem 78: 147-176.

Hidvegi T, Ewing M, Hale P, Dippold C, Beckett C, Kemp C, Maurice N, Mukherjee A, Goldbach C, Watkins S, et al. 2010. An autophagy-enhancing drug promotes degradation of mutant alpha 1-antitrypsin $Z$ and reduces hepatic fibrosis. Science 329: 229-232.

Hidvegi T, Mirnics K, Hale P, Ewing M, Beckett C, Perlmutter DH. 2007. Regulator of G Signaling 16 is a marker for the distinct endoplasmic reticulum stress state associated with aggregated mutant alpha 1 -antitrypsin $\mathrm{Z}$ in the classical form of alpha 1-antitrypsin deficiency. J Biol Chem 282: 27769-27780.

Hidvegi T, Schmidt BZ, Hale P, Perlmutter DH. 2005. Accumulation of mutant alpha 1-antitrypsin $\mathrm{Z}$ in the endoplasmic reticulum activates caspases- 4 and -12 , NFkappaB, and BAP31 but not the unfolded protein response. J Biol Chem 280: 39002-39015.

Hu C, Perlmutter DH. 2002. Cell-specific involvement of HNF-1beta in alpha (1)-antitrypsin gene expression in human respiratory epithelial cells. Am J Physiol Lung Cell Mol Physiol 282: L757-L765.

Huntington JA, Read RJ, Carrell RW. 2000. Structure of a serpin-protease complex shows inhibition by deformation. Nature 407: 923-926.

Inagi R, Nangaku M, Usuda N, Shimizu A, Onogi H, Izuhara Y, Nakazato K, Ueda Y, Oishi H, Takahashi S, et al. 2005. Novel serpinopathy in rat kidney and pancreas induced by overexpression of megsin. J Am Soc Nephrol 16: $1339-1349$.

Kamimoto T, Shoji S, Hidvegi T, Mizushima N, Umebayashi K, Perlmutter DH, Yoshimori T. 2006. Intracellular inclusions containing mutant alphal-antitrypsin $\mathrm{Z}$ are propagated in the absence of autophagic activity. J Biol Chem 281: 4467-4476.

Kruse KB, Brodsky JL, McCracken AA. 2006a. Characterization of an ERAD gene as VPS30/ATG6 reveals two alternative and functionally distinct protein quality control pathways: one for soluble $\mathrm{Z}$ variant of human alpha- 1 proteinase inhibitor (A1PiZ) and another for aggregates of A1PiZ. Mol Biol Cell 17: 203-312.

Kruse KB, Dear A, Kaltenbrun ER, Crum BE, George PM, Brennan SO, McCracken AA. 2006b. Mutant fibrinogen cleared from the endoplasmic reticulum via endoplasmic reticulum-associated protein degradation and autophagy: an explanation for liver disease. Am J Pathol 168: $1299-1308$.

Liao Y, Shikapwashya ON, Shteyer E, Dieckgraefe BK, Hruz PW, Rudnick DA. 2004. Delayed hepatocellular mitotic progression and impaired liver regeneration in early growth response-1-deficient mice. J Biol Chem 279: 43107-43116.

Lomas DA, Evans DL, Finch JT, Carrell RW. 1992. The mechanism of $\mathrm{Z}$ alpha 1-antitrypsin accumulation in the liver. Nature 357: 605-607.

Mahadeva R, Atkinson C, Li Z, Stewart S, Janciauskiene S, Kelley DG, Parmar J, Pitman R, Shapiro SD, Lomas DA. 2005. Polymers of $\mathrm{Z}$ alpha 1-antitrypsin co-localize with neutrophils in emphysematous alveoli and are chemotactic in vivo. Am J Pathol 166: 377-386.
Miranda E, MacLeod I, Davies MJ, Perez J, Romisch K, Crowther DC, Lomas DA. 2008. The intracellular accumulation of polymeric neuroserpin explains the severity of the dementia FENIB. Hum Mol Genet 17: 1527-1539.

Miranda E, Romisch K, Lomas DA. 2004. Mutants of neuroserpin that cause dementia accumulate as polymers within the endoplasmic reticulum. J Biol Chem 279: 28283-28291.

Miyata T, Inagi R, Nangaku M, Imasawa T, Sato M, Izuhara Y, Suzuki D, Yoshino A, Onogi H, Kimura M, et al. 2002. Overexpression of the serpin megsin induces progressive mesangial cell proliferation and expansion. J Clin Invest 109: 585-593.

Miyata T, Nangaku M, Suzuki D, Inagi R, Uragami K, Sakai H, Okubo K, Kurokawa K. 1998. A mesangium-predominant gene, megsin, is a new serpin up-regulated in $\operatorname{IgA}$ nephropathy. J Clin Invest 102: 828-836.

Pan S, Huang L, McPherson J, Muzny D, Rouhani F, Brantly M, Gibbs R, Sifers RN. 2009. Single nucleotide polymorphism-mediated translational suppression of endoplasmic reticulum mannosidase I modifies the onset of end-stage liver disease in alpha 1-antitrypsin deficiency. Hepatology 50: 275-281.

Perlmutter DH. 2002. Liver injury in alpha 1-antitrypsin deficiency: an aggregated protein induces mitochondrial injury. J Clin Invest 110: 1579-1583.

Perlmutter DH. 2006. The role of autophagy in alpha 1antitrypsin deficiency: a specific cellular response in genetic diseases associated with aggregation-prone proteins. Autophagy 2: 258-263.

Perlmutter DH, Brodsky JL, Balistreri WF, Trapnell BC. 2007. Molecular pathogenesis of alpha 1-antitrypsin deficiency-associated liver disease: a meeting review. Hepatology 45: 1313-1323.

Piitulainen E, Carlson J, Ohlsson K, Sveger T. 2005. Alpha 1-antitrypsin deficiency in 26-year-old subjects: lung, liver, and protease/protease inhibitor studies. Chest 128: $2076-2081$.

Poller W, Faber JP, Weidinger S, Tief K, Scholz S, Fischer M, Olek K, Kirchgesser M, Heidtmann HH. 1993. A leucine-to-proline substitution causes a defective alpha 1-antichymotrypsin allele associated with familial obstructive lung disease. Genomics 17: 740-743.

Qu D, Teckman JH, Omura S, Perlmutter DH. 1996. Degradation of a mutant secretory protein, alpha 1-antitrypsin $\mathrm{Z}$, in the endoplasmic reticulum requires proteasome activity. J Biol Chem 271: 22791-22795.

Rudnick DA, Liao Y, An JK, Muglia LJ, Perlmutter DH, Teckman JH. 2004. Analyses of hepatocellular proliferation in a mouse model of alpha 1-antitrypsin deficiency. Hepatology 39: 1048-1055.

Rudnick DA, Perlmutter DH. 2005. Alpha 1-antitrypsin deficiency: a new paradigm for hepatocellular carcinoma in genetic liver disease. Hepatology 42: 514-521.

Silverman GA, Whisstock JC, Bottomley SP, Huntington JA, Kaiserman D, Luke CJ, Pak SC, Reichhart JM, Bird PI. 2010. Serpins flex their muscle: I. Putting the clamps on proteolysis in diverse biological systems. J Biol Chem 285: 24299-24305.

Stein PE, Carrell RW. 1995. What do dysfunctional serpins tell us about molecular mobility and disease? Nat Struct Biol 2: 96-113. 
D.H. Perlmutter and G.A. Silverman

Suzuki D, Miyata T, Nangaku M, Takano H, Saotome N, Toyoda M, Mori Y, Zhang SY, Inagi R, Endoh M, et al. 1999. Expression of megsin mRNA, a novel mesangiumpredominant gene, in the renal tissues of various glomerular diseases. J Am Soc Nephrol 10: 2606-2613.

Takao M, Benson MD, Murrell JR, Yazaki M, Piccardo P, Unverzagt FW, Davis RL, Holohan PD, Lawrence DA, Richardson R, et al. 2000. Neuroserpin mutation S52R causes neuroserpin accumulation in neurons and is associated with progressive myoclonus epilepsy. J Neuropathol Exp Neurol 59: 1070-1086.

Teckman JH, An JK, Blomenkamp K, Schmidt B, Perlmutter D. 2004. Mitochondrial autophagy and injury in the liver in alpha 1-antitrypsin deficiency. Am J Physiol Gastrointest Liver Physiol 286: G851-G862.

Teckman JH, Burrows J, Hidvegi T, Schmidt B, Hale PD, Perlmutter DH. 2001. The proteasome participates in degradation of mutant alpha 1-antitrypsin $\mathrm{Z}$ in the endoplasmic reticulum of hepatoma-derived hepatocytes. J Biol Chem 276: 44865-44872.

Teckman JH, Gilmore R, Perlmutter DH. 2000. Role of ubiquitin in proteasomal degradation of mutant alpha (1)-antitrypsin Z in the endoplasmic reticulum. Am J Physiol Gastrointest Liver Physiol 278: G39-G48.

Teckman JH, Perlmutter DH. 2000. Retention of mutant alpha (1)-antitrypsin $\mathrm{Z}$ in endoplasmic reticulum is associated with an autophagic response. Am J Physiol Gastrointest Liver Physiol 279: G961-G974.

Verpy E, Couture-Tosi E, Eldering E, Lopez-Trascasa M, Spath P, Meo T, Tosi M. 1995. Crucial residues in the carboxy-terminal end of $\mathrm{C} 1$ inhibitor revealed by pathogenic mutants impaired in secretion or function. J Clin Invest 95: 350-359.

Villa P, Aznar J, Vaya A, Espana F, Ferrando F, Mira Y, Estelles A. 1999. Hereditary homozygous heparin cofactor II deficiency and the risk of developing venous thrombosis. Thromb Haemost 82: 1011-1014.

Werner ED, Brodsky JL, McCracken AA. 1996. Proteasomedependent endoplasmic reticulum-associated protein degradation: an unconventional route to a familiar fate. Proc Natl Acad Sci 93: 13797-13801.

Whisstock JC, Pike RN, Jin L, Skinner R, Pei XY, Carrell RW, Lesk AM. 2000. Conformational changes in serpins: II. The mechanism of activation of antithrombin by heparindagger. J Mol Biol 301: 1287-1305.

Whisstock JC, Silverman GA, Bird PI, Bottomley SP, Kaiserman D, Luke CJ, Pak SC, Reichhart JM, Huntington JA. 2010. Serpins flex their muscle: II. Structural insights into target peptidase recognition, polymerization, and transport functions. J Biol Chem 285: 2430724312.

Wu Y, Whitman I, Molmenti E, Moore K, Hippenmeyer P, Perlmutter DH. 1994. A lag in intracellular degradation of mutant alpha 1-antitrypsin correlates with the liver disease phenotype in homozygous PiZZ alpha 1antitrypsin deficiency. Proc Natl Acad Sci 91: 9014-9018.

Yamasaki M, Li W, Johnson DJ, Huntington JA. 2008. Crystal structure of a stable dimer reveals the molecular basis of serpin polymerization. Nature 455: 1255-1258.

Zhou A, Huntington JA, Carrell RW. 1999. Formation of the antithrombin heterodimer in vivo and the onset of thrombosis. Blood 94: 3388-3396. 


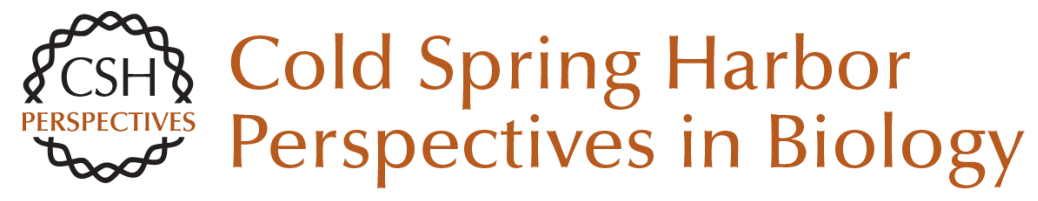

\title{
Hepatic Fibrosis and Carcinogenesis in $\alpha 1$-Antitrypsin Deficiency: A Prototype for Chronic Tissue Damage in Gain-of-Function Disorders
}

\author{
David H. Perlmutter and Gary A. Silverman
}

Cold Spring Harb Perspect Biol 2011; doi: 10.1101/cshperspect.a005801 originally published online January 12, 2011

\section{Subject Collection Protein Homeostasis \\ Proteome-Scale Mapping of Perturbed \\ Proteostasis in Living Cells \\ Isabel Lam, Erinc Hallacli and Vikram Khurana \\ Pharmacologic Approaches for Adapting \\ Proteostasis in the Secretory Pathway to \\ Ameliorate Protein Conformational Diseases Jeffery W. Kelly}

Cell-Nonautonomous Regulation of Proteostasis

in Aging and Disease

Richard I. Morimoto

The Autophagy Lysosomal Pathway and

Neurodegeneration

Steven Finkbeiner

Functional Modules of the Proteostasis Network Gopal G. Jayaraj, Mark S. Hipp and F. Ulrich Hartl

Protein Solubility Predictions Using the CamSol

Method in the Study of Protein Homeostasis Pietro Sormanni and Michele Vendruscolo

Recognition and Degradation of Mislocalized

Proteins in Health and Disease

Ramanujan S. Hegde and Eszter Zavodszky

The Nuclear and DNA-Associated Molecular Chaperone Network

Zlata Gvozdenov, Janhavi Kolhe and Brian C. Freeman
The Amyloid Phenomenon and Its Significance in

Biology and Medicine

Christopher M. Dobson, Tuomas P.J. Knowles and Michele Vendruscolo

A Chemical Biology Approach to the Chaperome

in Cancer--HSP90 and Beyond

Tony Taldone, Tai Wang, Anna Rodina, et al.

Proteostasis in Viral Infection: Unfolding the

Complex Virus-Chaperone Interplay

Ranen Aviner and Judith Frydman

The Proteasome and Its Network: Engineering for

Adaptability

Daniel Finley and Miguel A. Prado

Functional Amyloids

Daniel Otzen and Roland Riek

Chaperone Interactions at the Ribosome Elke Deuerling, Martin Gamerdinger and Stefan G. Kreft

Mechanisms of Small Heat Shock Proteins Maria K. Janowska, Hannah E.R. Baughman, Christopher N. Woods, et al.

Structure, Function, and Regulation of the Hsp90 Machinery

Maximilian M. Biebl and Johannes Buchner

For additional articles in this collection, see http://cshperspectives.cshlp.org/cgi/collection/

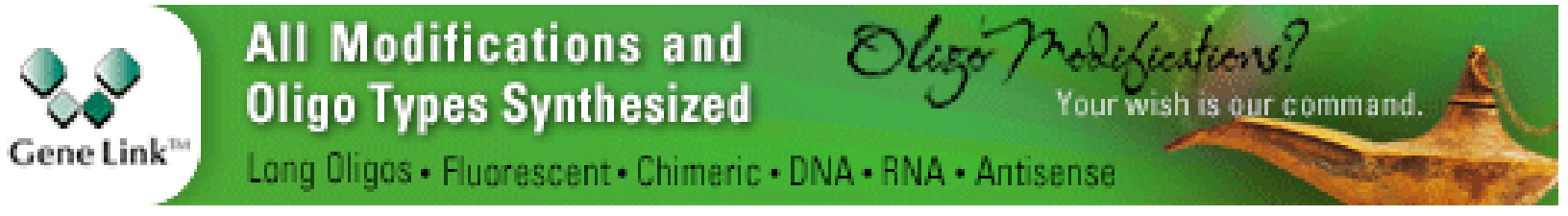


For additional articles in this collection, see http://cshperspectives.cshlp.org/cgi/collection/

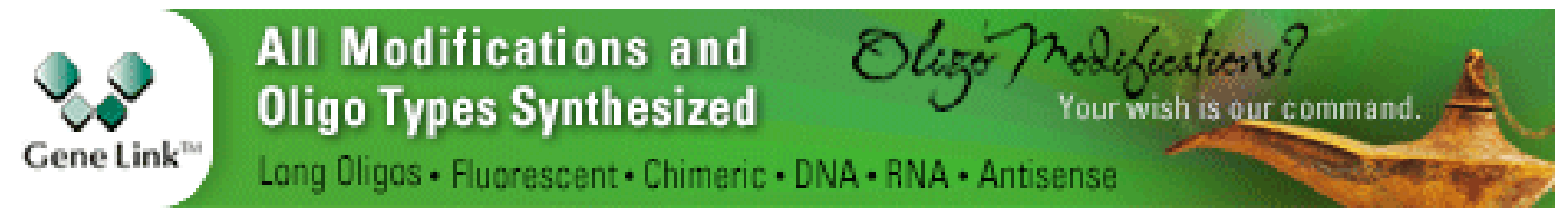

Copyright @ 2011 Cold Spring Harbor Laboratory Press; all rights reserved 